Proyecciones

Vol. 20, No 3, pp. 295-304, December 2001.

Universidad Católica del Norte

Antofagasta - Chile

\title{
DIFFERENTIABILITY OF SOLUTIONS OF THE ABSTRACT CAUCHY PROBLEM
}

\author{
HERNÁN R. HENRÍQUEZ * \\ Universidad de Santiago, Chile
}

\begin{abstract}
In this note we establish a result of differentiability for the mild solution of the inhomogeneous abstract Cauchy problem when the underlying space is reflexive.
\end{abstract}

2000 Mathematics Subject Classification : 47D09, 34G10.

Key Words and Phrases : Semigroup of Operators; Abstract Cauchy Problem; Differentiability of Solutions.

${ }^{*}$ This work was supported by DICYT-USACH, Project 04-9633HM. 


\section{Introduction.}

In this work we are concerned with regularity properties of solutions of the first order abstract Cauchy problem (in short, ACP). We refer the reader to $[3,10]$ for the theory of strongly continuous semigroup operators and the associated ACP.

Let $X$ be a Banach space endowed with a norm $\|\cdot\|$. Henceforth $T(t)$ is a strongly continuous semigroup of operators on $X$ with infinitesimal generator $A$.

The existence of solutions of the first order abstract Cauchy problem

$$
\begin{aligned}
x^{\prime}(t) & =A x(t)+h(t), 0 \leq t \leq a, \\
x(0) & =x_{0},
\end{aligned}
$$

it has been treated in several works. We only mention here the texts $[3,10]$ and the references cited therein. Similarly, the existence of solutions of the semilinear abstract Cauchy problem it has been discussed in $[1,9]$.

Assuming that $h:[0, a] \rightarrow X$ is integrable the function given by

$$
x(t)=T(t) x_{0}+\int_{0}^{t} T(t-s) h(s) d s, \quad 0 \leq t \leq a,
$$

is said mild solution of (1.1)-(1.2). In the case in which $h$ is continuous , the function $x(\cdot)$ is called a classic solution on $[0, a]$ of $(1.1)-(1.2)$ if $x$ is a function of class $C^{1}, x(t) \in D(A)$ and (1.1) is verified.

The existence of classical solutions of (1.1)-(1.2) as well as some weaker forms of differentiability of solutions have been studied in a number of works. We refer the reader to $[2,8,10,12,13,14]$ and the references therein indicated.

The purpose of this note is to establish a new condition in order to the mild solution $x(\cdot)$ turn to be a classical solution .

Next the notation $C([0, a] ; X)$ stands for the space of continuous functions from $[0, a]$ into $X$, whilst $B V([0, a] ; X)$ represents the space of functions with bounded variation from $[0, a]$ into $X$. For a function $h \in B V([0, a] ; X)$ we denote by $V(h)$ the variation of $h$ on $[0, a]$ and by $v(t, h)$ the variation of $h$ on $[0, t]$, for $0 \leq t \leq a$. Additional termi- 
nology and notations are those generally used in functional analysis. In particular, $X^{*}$ denotes the dual space of $X$.

\section{Results.}

In this section $h$ denotes a continuous function of bounded variation on a fixed interval $[0, a], a>0$. We define the translation of $h$ by

$$
\mathcal{T}_{t} h(s)=\left\{\begin{aligned}
h(s+t), & s \leq a-t, \\
h(a), & s \geq a-t
\end{aligned}\right.
$$

for $t \geq 0$. Let $\mu(t, h)=V\left(\mathcal{T}_{t} h-h\right)$.

We introduce the following condition for a function $h \in C([0, a] ; X) \cap$ $B V([0, a] ; X)$.

$\left(H_{0}\right) \quad \mu(t, h) \rightarrow 0$, as $t \rightarrow 0^{+}$.

Initially we discuss some examples.

Example 1. If $h \in W^{1,1}([0, a] ; X)$, then $\mu(t, h) \rightarrow 0, t \rightarrow 0^{+}$.

Example 2. Let $h:[0,1] \rightarrow \mathbb{R}$ be the function defined in [4], Exercise 4.19. Let $E$ be a perfect nowhere dense set with measure 0 included in $[0,1]$. Let $\left(a_{k}, b_{k}\right), k \in \mathbb{N}$, be disjoint intervals such that $(0,1) \backslash E=\bigcup_{k=1}^{\infty}\left(a_{k}, b_{k}\right)$ and let $\sum_{k=1}^{\infty} c_{k}$ be a convergent series of positive number with sum equal to 1 . For each $x \in[0,1]$ let

$$
I(x)=\left\{k:\left[a_{k}, b_{k}\right] \cap[0, x] \neq \Phi\right\}
$$

and define

$$
h(x)=\sum_{k \in I(x)} c_{k}
$$

It is clear that $h(0)=0, h(1)=1$. Moreover, $h$ is continuous and nondecreasing with $h^{\prime}=0$, a.e. Thus $h$ is a singular function . Now we establish that $h$ does not satisfy $\left(H_{0}\right)$. In fact, from (2.1) it follows easily that for each $t>0$ and $0 \leq s \leq 1-t$,

$$
h(s+t)-h(s)=\sum_{k \in I} c_{k},
$$


where $I=\left\{k \in \mathbb{N}: a_{k} \in(s, s+t]\right\}$. In addition, for $n \in \mathbb{N}$ we can choose $t>0$ small enough such that $\bigcup_{i=1}^{n}\left[a_{i}, b_{i}\right] \subseteq[0,1-t], a_{i}+3 t<$ $b_{i}$, and, for each $k=1, \cdots, n, a_{k}-t \notin \bigcup_{i=1, i \neq k}^{n}\left[a_{i}, b_{i}\right]$.

Defining $\alpha_{i}=a_{i}-t / 2$ and $\beta_{i}=a_{i}+2 t$ it follows from (2.2) that $h\left(\alpha_{i}+t\right)-h\left(\alpha_{i}\right)=c_{i}$ and $h\left(\beta_{i}+t\right)-h\left(\beta_{i}\right)=0$. From this we obtain that

$$
\begin{aligned}
V\left(\mathcal{T}_{t} h-h\right) & \geq \sum_{i=1}^{n}\left|\left(\mathcal{T}_{t} h-h\right)\left(\beta_{i}\right)-\left(\mathcal{T}_{t} h-h\right)\left(\alpha_{i}\right)\right| \\
& =\sum_{i=1}^{n}\left|h\left(\beta_{i}+t\right)-h\left(\beta_{i}\right)-\left(h\left(\alpha_{i}+t\right)-h\left(\alpha_{i}\right)\right)\right| \\
& =\sum_{i=1}^{n} c_{i}
\end{aligned}
$$

which implies that $\mu(t, h)$ does not converge to 0 as $t \rightarrow 0^{+}$.

Example 3. Let $h:[0,1] \rightarrow \mathbb{R}$ be the singular function defined in [6], Example 18.8. As above, $h(0)=0, h(1)=1, h$ is continuous and strictly increasing and $h^{\prime}=0$, a.e. We will show that this function satisfies the assumption $\left(H_{0}\right)$. Initially, for completeness we include here the construction carried out in [6].

Let $\left(t_{n}\right)_{n}$ be a sequence in $(0,1)$. Set $F_{1}(0)=0, F_{1}(1)=1$, $F_{1}\left(\frac{1}{2}\right)=\frac{1+t_{1}}{2}$ and define $F_{1}$ to be linear on $\left[0, \frac{1}{2}\right]$ and $\left[\frac{1}{2}, 1\right]$. Suppose that $F_{1}, F_{2}, \cdots, F_{n}$ have been defined. Then define

$$
\begin{gathered}
F_{n+1}\left(\frac{k}{2^{n}}\right)=F_{n}\left(\frac{k}{2^{n}}\right), \text { for } k=0,1, \cdots, 2^{n}, \\
\mathrm{~F}_{n+1}\left(\frac{2 k+1}{2^{n+1}}\right)=\frac{1-t_{n+1}}{2} F_{n}\left(\frac{k}{2^{n}}\right)+\frac{1+t_{n+1}}{2} F_{n}\left(\frac{k+1}{2^{n}}\right),
\end{gathered}
$$

for $k=0,1, \cdots, 2^{n}-1$,

and complete the definition of $F_{n+1}$ as a continuous linear function in the intervals $\left[\frac{k}{2^{n+1}}, \frac{k+1}{2^{n+1}}\right]$, for $k=0,1, \cdots, 2^{n+1}-1$. It 
is shown in [6] that $\left(F_{n}\right)_{n}$ is a nondecreasing sequence. Thus this sequence converges to a function $h$ which satisfies the properties already mentioned. Applying now the Dini's theorem ([11]) we obtain that the convergence of $\left(F_{n}\right)_{n}$ is uniform. Since $\left(\mathcal{T}_{t} F_{n}-F_{n}\right)_{n}$ converges uniformly to $\mathcal{T}_{t} h-h$, as $n \rightarrow \infty$, and this convergence is also uniform on $t \geq 0$, it is follows that $\mu\left(t, F_{n}\right) \rightarrow \mu(t, h), n \rightarrow \infty$, and this convergence is uniform on $t$. In view of $F_{n}$ is absolutely continuous, from Example 1 we infer that $\mu\left(t, F_{n}\right) \rightarrow 0$, as $t \rightarrow 0^{+}$, which implies that $\mu(t, h) \rightarrow 0$, as $t \rightarrow 0^{+}$.

To study the regularity of solutions of the abstract Cauchy problem (1.1)-(1.2) we begin by establishing some preliminary lemmas.

In the sequel we denote by $M$ a positive constant such that $\|T(t)\| \leq$ $M, 0 \leq t \leq a$. Moreover, for a fixed $h$, we use the notation

$$
u(t)=\int_{0}^{t} T(t-s) h(s) d s,
$$

Lemma 2.1. Assume that $X$ is a reflexive space . Let $T(\cdot)$ be a strongly continuous semigroup of operators on $X$ and let $h:[0, a] \rightarrow$ $X$ be a continuous function of bounded variation which satisfies the assumption $\left(H_{0}\right)$. Then the Riemann-Stieltjes integral

$$
w(t)=\int_{0}^{t} T(t-s) d_{s} h=\int_{0}^{t} T(s) d_{s} h(t-s)
$$

exists in the weak topology and define a continuous function $w$ : $[0, a] \rightarrow X$.

Proof. $\quad$ Let $\Lambda: X^{*} \rightarrow \mathbb{C}$ be defined by

$$
\Lambda\left(x^{*}\right)=\int_{0}^{t}<T(t-s)^{*} x^{*}, d_{s} h>.
$$

The Riemann-Stieltjes integral in the above expression exists because $T(\cdot)^{*} x^{*}$ is a continuous function $([10])$ and $h$ has bounded variation $([7])$. Moreover, $\Lambda$ is linear and

$$
\left|\Lambda\left(x^{*}\right)\right| \leq M\left\|x^{*}\right\| V(h)
$$

Consequently, $\Lambda \in X^{* *}$ and in view of that $X$ is reflexive we infer the existence of $w(t) \in X$ such that $\Lambda\left(x^{*}\right)=<x^{*}, w(t)>$, for all $x^{*} \in X^{*}$. 
On the other hand, for $t<1$ and $\tau$ small enough, from the relations

$$
\begin{aligned}
w(t+\tau)-w(t)= & \int_{0}^{t+\tau} T(s) d_{s} h(t+\tau-s)-\int_{0}^{t} T(s) d_{s} h(t-s) \\
= & \int_{0}^{t} T(t-s) d_{s}[h(\tau+s)-h(s)] \\
& +\int_{0}^{\tau} T(t+s) d_{s} h(\tau-s)
\end{aligned}
$$

we deduce that

$$
\|w(t+\tau)-w(t)\| \leq M \mu(\tau, h)+M v(\tau, h) .
$$

Since $\mu(\tau, h) \rightarrow 0, \tau \rightarrow 0$, because the condition $\left(H_{0}\right)$ holds and $v(\tau, h) \rightarrow 0, \tau \rightarrow 0$, by the Proposition I.2.9 in [7]) the previous estimation shows that $w(\cdot)$ is right continuous at $t$. Similarly, one can prove that $w$ is left continuous at $t>0$.

Next we denote by $\chi_{E}$ the characteristic function of a set $E$.

Lemma 2.2. Let $h:[0, a] \rightarrow X$ be the step function $h=\sum_{i=1}^{n} x_{i} \chi_{I_{i}}$, where $I_{i}$ are intervals and $\left\{I_{1}, \cdots, I_{n}\right\}$ is a partition of $[0, a]$. Then the function $u$ given by (2.3) is piecewise smooth, $u(t) \in D(A), A u(\cdot)$ is continuous on $[0, a]$ and $u^{\prime}(t)=A u(t)+h(t), t \notin P$, where $P$ denotes the set formed by the extreme points of intervals $I_{i}, i=i, \cdots, n$.

Proof. Applying the linearity of $u$ in terms of $h$, it is sufficient to prove the assertion for a function $h=x \chi_{I}$ where $I$ is an interval of type $\left[t_{1}, t_{2}\right]$. In fact, in this case, $u(t)$ is given by

$$
u(t)=\left\{\begin{aligned}
0, & 0 \leq t \leq t_{1}, \\
\int_{0}^{t-t_{1}} T(s) x d s, & t_{1} \leq t \leq t_{2} \\
\int_{t-t_{2}}^{t-t_{1}} T(s) x d s, & t_{2} \leq t .
\end{aligned}\right.
$$


From the properties of semigroups we infer that $u(t) \in D(A)$ and that

$$
A u(t)=\left\{\begin{aligned}
0, & 0 \leq t \leq t_{1}, \\
T\left(t-t_{1}\right) x-x, & t_{1} \leq t \leq t_{2}, \\
T\left(t-t_{1}\right) x-T\left(t-t_{2}\right) x, & t_{2} \leq t .
\end{aligned}\right.
$$

This shows that $A u(\cdot)$ is continuous . Moreover, it is immediate to verify that $u^{\prime}(t)=A u(t)+h(t), t \neq t_{1}, t_{2}$.

Now we can prove the main result of this note.

Theorem 2.1. Assume that $X$ is a reflexive space and let $h$ be a continuous function of bounded variation on $[0, a]$ which satisfies assumption $\left(H_{0}\right)$. Let $x_{0} \in D(A)$. Then the mild solution of (1.1)-(1.2) is a classical solution .

Proof. We consider a sequence $\left(h_{n}\right)_{n}$ of step functions, where each $h_{n}$ is given by

$$
h_{n}=\sum_{i=1}^{n} h\left(t_{i}\right) \chi_{I_{i}} .
$$

In this expression we have denoted $I_{i}=\left[t_{i-1}, t_{i}\right), i=1, \cdots, n-1$, and $I_{n}=\left[t_{n-1}, t_{n}\right]$, where the points $t_{i}$ have been chosen as $t_{i}=\frac{a}{n} i, \quad i=$ $0,1, \cdots, n$.

It is clear that the sequence $\left(h_{n}\right)_{n}$ converge uniformly to $h$. Let $u_{n}$ be the function given by (2.3), with $h_{n}$ instead of $h$. Then, $u_{n} \rightarrow$ $u, n \rightarrow \infty$, uniformly on $[0, a]$. Moreover, by Lemma 2.2 we have that $u_{n}(t) \in D(A)$ and, if we fix $0 \leq t \leq a$ and $n \in \mathbb{N}$, then $t \in I_{k}$, for some $k=1, \cdots, n$. From our definitions we can write

$$
\begin{aligned}
A u_{n}(t) & =A \sum_{i=1}^{k-1} \int_{t_{i-1}}^{t_{i}} T(t-s) h\left(t_{i}\right) d s+A \int_{t_{k-1}}^{t} T(t-s) h\left(t_{k}\right) d s \\
& =\sum_{i=1}^{k-1}\left[T\left(t-t_{i-1}\right)-T\left(t-t_{i}\right)\right] h\left(t_{i}\right)+\left[T\left(t-t_{k-1}\right)-I\right] h\left(t_{k}\right) \\
& =\sum_{i=1}^{k-1} T\left(t-t_{i-1}\right)\left[h\left(t_{i}\right)-h\left(t_{i-1}\right)\right]+T\left(t-t_{k-1}\right)
\end{aligned}
$$




$$
\begin{aligned}
& {\left[h(t)-h\left(t_{k-1}\right)\right]+T\left(t-t_{k-1}\right)\left[h\left(t_{k}\right)-h(t)\right]+} \\
& T(t) h(0)-h\left(t_{k}\right)
\end{aligned}
$$

so that

$$
\left\|A u_{n}(t)\right\| \leq M V(h)+(M+1)\|h\|_{\infty} .
$$

This shows that $\left(A u_{n}(t)\right)_{n}$ is a bounded sequence. Consequently, there is a subsequence which converges to $z(t) \in X$ in the weak topology. Moreover, from (2.4) it follows that

$$
z(t)=w(t)-h(t)+T(t) h(0) .
$$

An standard argument shows that the full sequence $\left(A u_{n}(t)\right)_{n}$ converges to $w(t)$. As $A$ is a closed operator this implies that $u(t) \in D(A)$ and $z(t)=A u(t)$.

An application of Lemma 2.1 yields that $A u(\cdot)$ is a continuous function. On the other hand, from Lemma 2.2 we have

$$
u_{n}^{\prime}(t)=A u_{n}(t)+h_{n}(t), \quad n \in \mathbb{N}, \quad t \neq i / n, i=1, \cdots, n-1,
$$

so that for each $x^{*} \in X^{*}$ we obtain

$$
<x^{*}, u_{n}(t)>=\int_{0}^{t}<x^{*}, A u_{n}(s)+h_{n}(s)>d s
$$

and taking limit as $n \rightarrow \infty$, it follows that

$$
<x^{*}, u(t)>=\int_{0}^{t}<x^{*}, A u(s)+h(s)>d s
$$

which implies that

$$
u(t)=\int_{0}^{t} A u(s) d s+\int_{0}^{t} h(s) d s .
$$

This shows that $u(\cdot)$ is a function of class $C^{1}$ that satisfies (1.1)-(1.2).

A similar result holds for the second order abstract Cauchy prob$\operatorname{lem}([5])$. 


\section{References}

[1] Cazenave, T. and A. Haraux, An Introduction to Semilinear Evolution Equations. Oxford University Press, Oxford, 1998.

[2] Eidelman, Y. S. and I. V. Tichonov, A Note on the Abstract Cauchy Problem, Semigroup Forum, 54(1997), 112-116.

[3] Engel, K-J. and R. Nagel, One-parameter Semigroups for Linear Evolution Equations. Springer-Verlag, New York, 2000.

[4] Gordon, R. A., The integrals of Lebesgue, Denjoy, Perron, and Henstock. Amer. Math. Soc., Providence, 1994.

[5] Henríquez, H. R. and C. Vásquez, Differentiability of Solutions of the Second Order Abstract Cauchy Problem. To appear in Semigroup Forum.

[6] Hewitt, E. and K. Stromberg, Real and Abstract Analysis. Springer-Verlag, Berlin, 1969.

[7] Hönig, C. S., The Abstract Riemann-Stieltjes Integral and its Applications to Linear Differential Equations with Generalized Boundary Conditions. Notas do Instituto de Matemática e Estatística da Universidade de São Paulo. Série Matemática $\mathrm{N}^{\circ} 1$, 1973.

[8] Hönig, C. S., Semigroups and Semivariation, $14^{\circ}$ Seminário Brasileiro de Análise, 1981, 185-193.

[9] Miyadera, I., Nonlinear Semigroups . Amer. Math. Soc, Providence, 1992.

[10] Pazy, A., Semigroups of Linear Operators and Applications to Partial Differential Equations. Springer-Verlag, New York, 1983.

[11] Ray, W. O., Real Analysis. Prentice Hall, Englewood Cliffs, 1988. 
[12] Travis, C. C., Differentiability of weak solutions to an abstract inhomogeneous differential equation, Proc. Amer. Math. Soc. 82 (1981), 425-430.

[13] Webb, G. F., Regularity of Solutions to an Abstract Inhomogeneous Linear Differential Equations. Proc. Amer. Math. Soc. 62 (1977), 271-277.

[14] Zaidman, S. D., Abstract Differential Equations. Research Notes in Mathematics, 36, Pitman, San Francisco, 1979.

Received : May, 2001.

\section{Hernán R. Henríquez Miranda}

Departamento de Matemática

Universidad de Santiago de Chile

Casilla 307

Correo 2

Santiago

Chile

E-mail: henrique@fermat.usach.cl 